\title{
The effects of probiotic supplementation on selected cognitive functions in young women. Preliminary data.
}

\author{
Dominik Czajeczny, Karolina Kabzińska, Ewa Mojs, Rafał Wojciech Wójciak \\ Department of Clinical Psychology, Poznan University of Medical Sciences \\ email: d.czajeczny@gmail.com
}

Background and aim

A growing body of research shows promising results on the health benefits of probiotics in clinical samples. Reported data shows positive effects of probiotics on mood (Benton, Williams, \& Brown, 2007; Steenbergen, Sellaro, van Hemert, Bosch, \& Colzato, 2015), regulation of HPA axis activity (Schmidt et al., 2015) and cognitive functioning (Akbari et al., 2016; Rudzki et al., 2019). Probiotics have become increasingly popular pharmacy and grocery items, in the form of supplements and functional foods, such as yogurt and other fermented products (Adams, 2015). The question posed by this study is whether prophylactic intake of probiotic bacteria is beneficial for psychological functioning of healthy adults?

\section{Results}

- No significant differences between groups were found at pretest.

- Both groups showed decreased RT in Lexical Decisions Task, in both conditions.

- The placebo group showed increased word count in Verbal Fluency Task and Verbal Memory Test.

- The supplementation group showed no improvement in Verbal Fluency Task and Verbal Memory Test.

- Probiotic supplementation might be beneficial for health by serving as a protective factor.

\section{Probiotic supplementation shows no benefits for cognitive functioning over placebo in healthy young women.}

\section{Materials and methods}

40 women aged 20-30 volunteered. Participants after antibiotic treatment (up to 3 months prior to assessment), with psychiatric disorders and bowel function disorders were excluded. Participants were randomly assigned to groups $(n=20$ in both groups) and were taking provided capsules for 6 weeks.

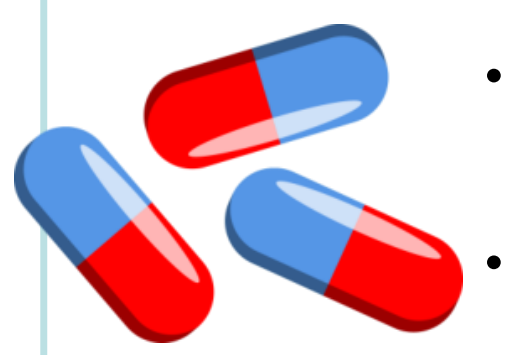

\section{Probiotic supplement (Bifidobacterium} lactis BS01, Lactobacillus acidophilus LA02

Placebo (maltodextrin)

A modified version of Verbal Fluency Task FAS was used to induce stress. Participants were asked to generate words aloud for 1 minute, for each letter (F-A-S), in the presence of the experimenter.

Verbal Memory Test: 10 words over 5 trials

- Lexical Decisions Task: stimuli were preceded by either neutral or affective pictures, taken from GAPED (DanGlauser \& Scherer, 2011). Participants were asked to respond ' 1 ' for word and ' 2 ' nonword, using their dominant hand on numerical keyboard.

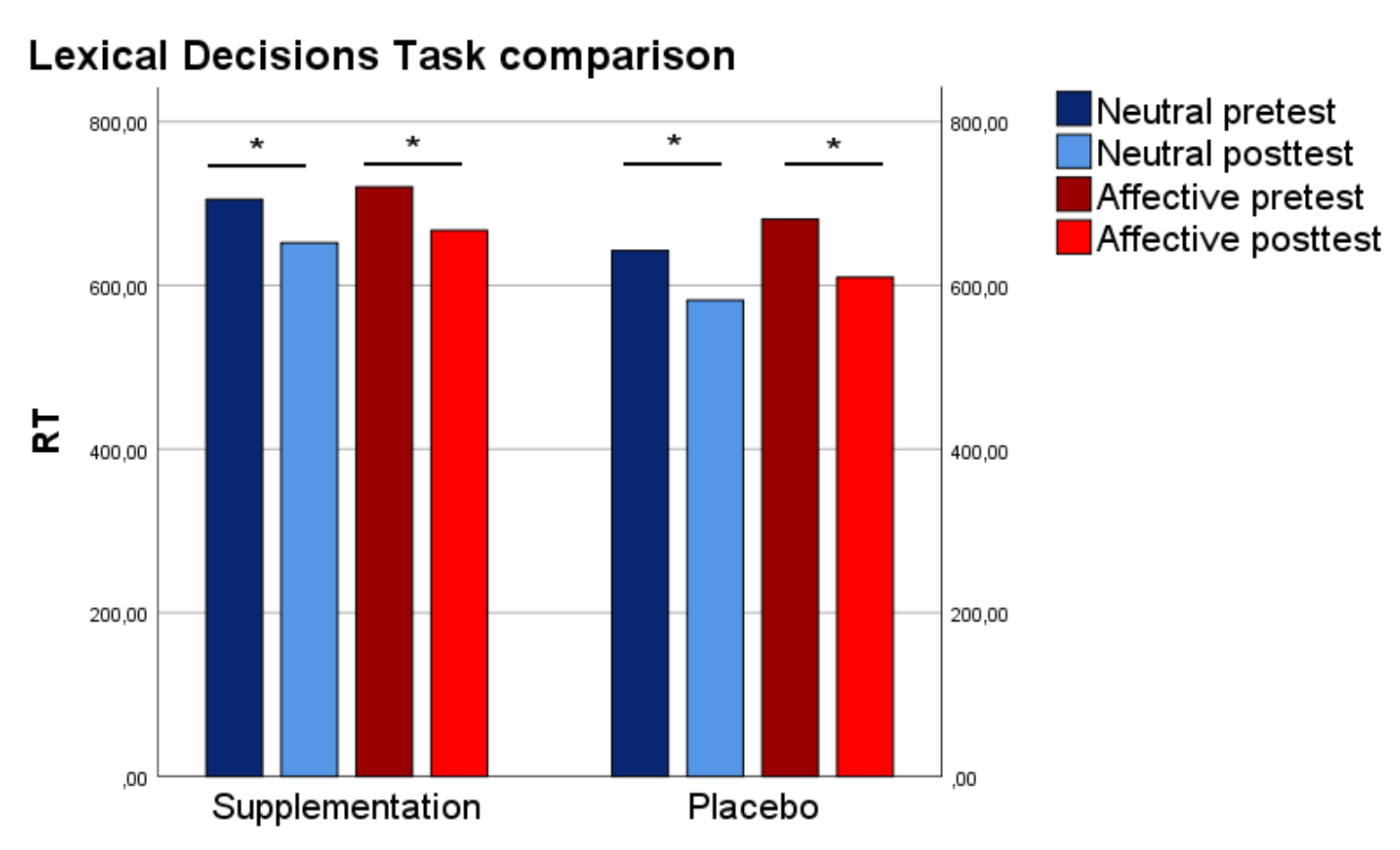

* represents $p<0.05$
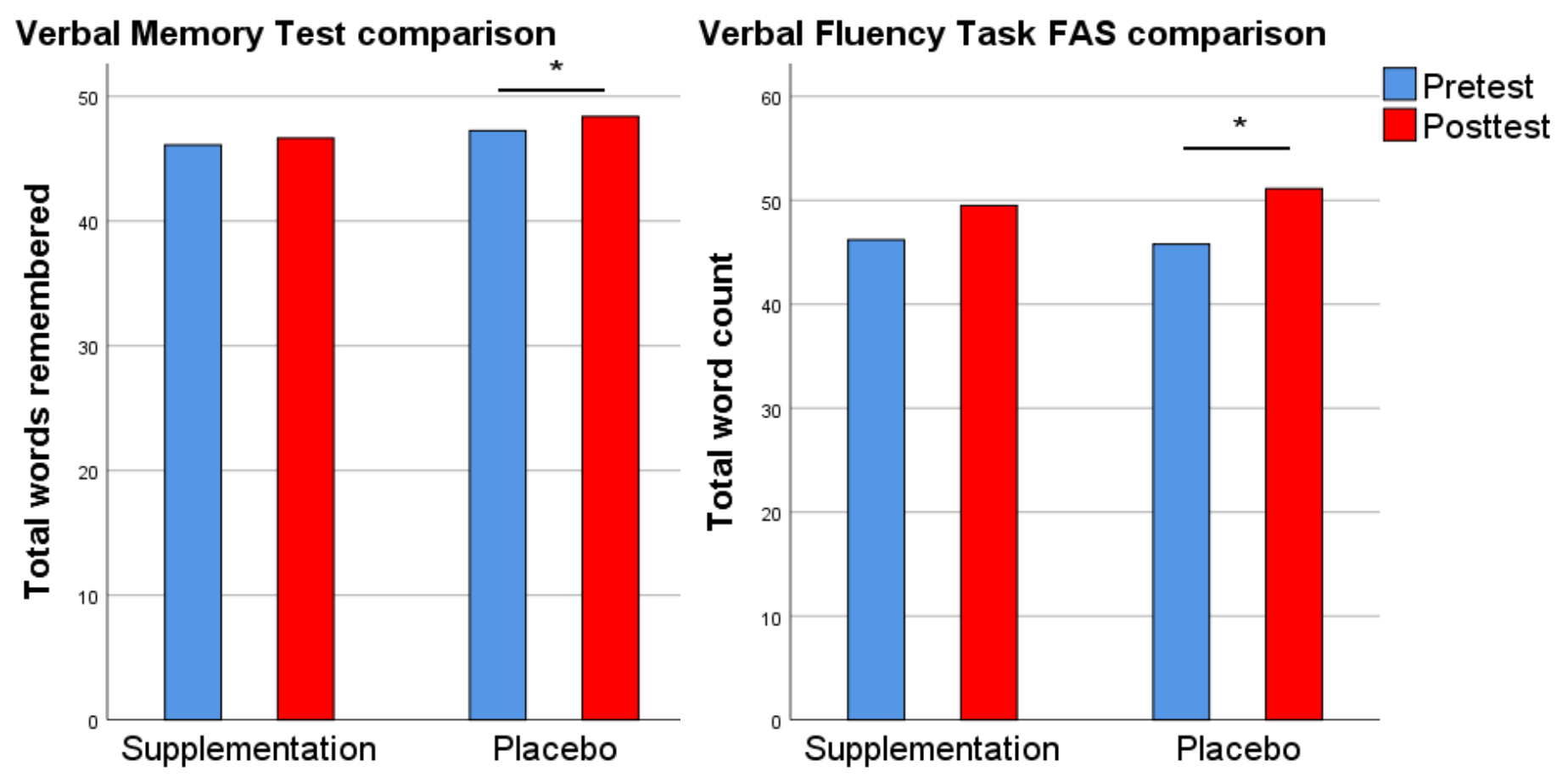

* represents $p<0.05$

\section{Limitations}

- Small sample size

- Relative low sensitivity of the applied measures

- Study not controlling for diet and GI tract functioning

Check

references

using QR code

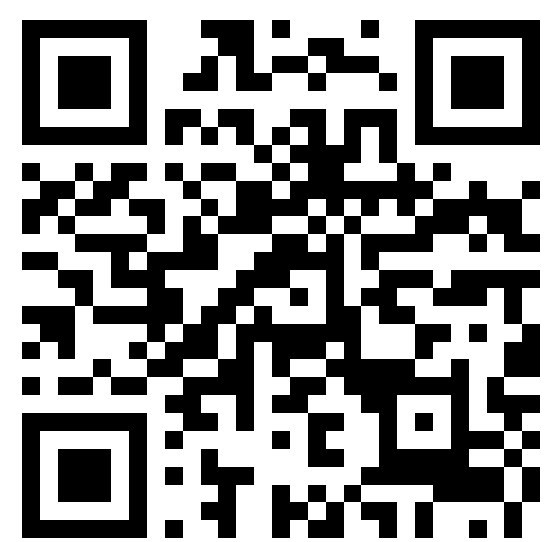

